\title{
Combined comparative genomic hybridization and transcriptomic analyses of ovarian granulosa cell tumors point to novel candidate driver genes
}

\author{
Sandrine Caburet ${ }^{1,2,6^{*}}$, Mikko Anttonen ${ }^{3,4}$, Anne-Laure Todeschini ${ }^{1,2}$, Leila Unkila-Kallio ${ }^{3}$, Denis Mestivier ${ }^{1,2}$,
} Ralf Butzow ${ }^{3,5}$ and Reiner A Veitia ${ }^{1,2,6^{*}}$

\begin{abstract}
Background: Ovarian granulosa cell tumors (GCTs) are the most frequent sex cord-stromal tumors. Several studies have shown that a somatic mutation leading to a C134W substitution in the transcription factor FOXL2 appears in more than 95\% of adult-type GCTs. Its pervasive presence suggests that FOXL2 is the main cancer driver gene. However, other mutations and genomic changes might also contribute to tumor formation and/or progression.

Methods: We have performed a combined comparative genomic hybridization and transcriptomic analyses of 10 adult-type GCTs to obtain a picture of the genomic landscape of this cancer type and to identify new candidate co-driver genes.

Results: Our results, along with a review of previous molecular studies, show the existence of highly recurrent chromosomal imbalances (especially, trisomy 14 and monosomy 22) and preferential co-occurrences (i.e. trisomy 14/monosomy 22 and trisomy 7/monosomy 16q). In-depth analyses showed the presence of recurrently broken, amplified/duplicated or deleted genes. Many of these genes, such as AKT1, RUNX1 and LIMA1, are known to be involved in cancer and related processes. Further genomic explorations suggest that they are functionally related.

Conclusions: Our combined analysis identifies potential candidate genes, whose alterations might contribute to adult-type GCT formation/progression together with the recurrent FOXL2 somatic mutation.
\end{abstract}

Keywords: Ovarian granulosa cell tumor, Driver genes, CGH, Transcriptomics

\section{Background}

Ovarian granulosa cell tumors (GCTs) are the most frequent sex cord-stromal tumors, and account for more than $5 \%$ of ovarian cancers [1]. Two different forms, juvenile and adult, have been described based on the age of onset and histopathological features [2]. GCTs tend to be low-grade malignancies, but can recur up to 40 years after primary tumor resection [3]. Various studies have revealed that a somatic mutation leading to the p.C134W substitution in the transcription factor FOXL2 appears in $>95 \%$ of adult-type GCTs [4].

Transactivation studies have suggested that the p.C134W mutation could perturb the functional interaction between

\footnotetext{
*Correspondence: caburet.sandrine@ijm.univ-paris-diderot.fr; veitia.reiner@ ijm.univ-paris-diderot.fr

${ }^{1}$ Institut Jacques Monod, Paris, France

Full list of author information is available at the end of the article
}

FOXL2 with SMAD3 [5] and FOXL2 activity in other systems [6]. This variant is also deficient in its ability to promote apoptosis [7] and displays a mild loss-of-function on targets involved in cell cycle and DNA-damage repair [8].

We have recently performed a transcriptomic profiling of 10 human adult-GCTs and ethnically-matched GC controls. This study showed that GCTs display several typical hallmarks of cancer. For instance, among FOXL2 direct targets, we detected an up-regulation of genes associated with cell cycle control and a down-regulation of genes related with apoptosis [9]. The pervasive somatic FOXL2 mutation is expected to be the main driver of GCTs. However, we hypothesize that it might engender or be accompanied by other mutations and genomic changes that might facilitate tumor formation and/or progression. Here, we have explored this possibility by performing a comparative genomic hybridization (CGH) 
analysis of the aforementioned tumor samples in correlation with their transcriptomes. This combined analysis is the first attempt to obtain a "bird's eye" view of the genomic landscape of this cancer type and to identify new candidate (co-)driver genes (termed henceforth driver genes for simplicity).

\section{Methods}

\section{Ethics statement}

This research involves human samples and has been performed with the approval of the Ethics Committee of the Helsinki University Central Hospital. Research was carried out in compliance with the Helsinki Declaration.

\section{Comparative Genomic Hybridization (CGH)}

The CGH was performed using genomic DNA from the tumor samples co-hybridized with an equimolar mix of 10 ethnically-matched (finnish) DNA samples on NimbleGen 12x135K CGH arrays, which 60-mer probes spaced every $13 \mathrm{~kb}$ on average. Sample processing, hybridization and data acquisition were performed at Nimblegen according to an in-house standard protocol. CGH microarray data are available in the ArrayExpress database (www.ebi.ac.uk/arrayexpress) under accession number E-MTAB-2873. CGH data were analyzed as $\log 2$ values of the ratio between the fluorescences of tumor and reference genomic DNA samples, using MeV software (TM4 suite, http://www.tm4.org).

\section{CGH and transcriptome correlations}

For large-scale alterations, the $\mathrm{CGH}$ data were averaged for sliding windows of $130 \mathrm{~kb}$ over the relevant chromosomes. For the transcriptomic data, we used our previously published data from the 10 tumors, as described in [9] (NimbleGen Human Expression $12 \times$ $135 \mathrm{~K}$ array set, accession E-MTAB-483 in the ArrayExpress database). The two independent transcriptomic hybridizations were averaged for each transcript, and then we computed the average expression levels for each gene.

To better measure the impact of large-scale genomic alterations on gene expression we divided the expression values for genes located within aneuploid regions by their mean expression in the tumors without the analyzed alteration. Expression ratios above/ below 1 in the natural scale (or above/below 0 in $\log 2$ scale) in aneuploid regions are suggestive of a "correlation" between genomic duplications/deletions and gene expression. Finally, these ratios were averaged for 30 windows (of equal size) per chromosome. The $\mathrm{CGH}$ and transcriptomic profiles were displayed using MeV software.
To identify candidate drivers, we used the combination of several criteria. First, we aimed at identify genes with an expression correlated to small-scale imbalances. For this, the CGH probes, amplified/duplicated or deleted in at least $50 \%$ of the cells (which corresponds to log ratio of 0,322 or $-0,415$, respectively) and in at least two tumors, were identified using $\mathrm{MeV}$ software. Next, genes were retained for further analysis if one or several amplified/deleted CGH probe(s) mapped within $25 \mathrm{~kb}$ of the gene coordinates. Given the $13 \mathrm{~kb}$-resolution of the CGH chip, a 25-kb maximum spacing enabled us to detect all relevant genes. Furthermore, transcriptomic values had to be significantly correlated with the $\mathrm{CGH}$ data over all the tumors (Pearson correlation coefficient, R). The threshold of statistical significance used for $\mathrm{R}$ was determined considering that, for a sample size $\mathbf{N}$, with observed values of $\mathbf{R}$, there is a statistic $\mathrm{t}$ such that:

$$
t=R \sqrt{\frac{n-2}{1-R^{2}}}
$$

which follows approximately a Student-t distribution with $\mathbf{N}-2$ degrees of freedom. Application of this formula to any particular observed value of $\mathbf{R}$ will test the null hypothesis that the observed value comes from a population in which the correlation between the two variables is 0 . For a sample size of $\mathrm{N}=10$ (all tumors in our cohort), the $\mathrm{R}$ that can be considered as statistically significant according to this test is 0.63 . In order to exclude any effect of large-scale imbalances (such as trisomies or monosomies), the gene-centered CGH/expression correlations were computed in the relevant genomic regions only for tumors without the large-scale imbalances. Therefore, we adjusted the threshold for $\mathrm{R}$ significance accordingly, to $\mathrm{R}>=0.67$ for 9 samples, $\mathrm{R}>=0.71$ for 8 samples and $R>=0.76$ for 7 samples. Due to the small sample size of our cohort, we could not apply a correction for multiple testing, such as Bonferroni's or Benjamini-Hochberg's. Candidate driver genes were further selected when i) being completely included in the genomic alteration (i.e. fully amplified or deleted) and ii) not being included in frequent CNVs in healthy individuals, as defined by the Database of Genomic Variants (DGV, http://dgv.tcag.ca/dgv/app/home, as of 31/05/ 2013).

Recurrently broken genes were identified by the existence, in at least 2 tumors, of one or several closely mapping breakpoints defined by amplifications/deletions upstream and downstream, within the relevant gene. We excluded genes for which the breakpoints mapped near or within frequent CNVs according to DGV. This step was necessary because the control DNA used for CGH 
was a pool of ethnically-matching DNA samples, and not the somatic DNA from the respective patients.

\section{Expressional correlation, protein interactor sharing and transcriptomic neighbors sharing between candidate drivers}

Hierarchical clustering of the expression levels of broken, amplified or deleted candidate drivers and FOXL2 was performed with the $\mathrm{MeV}$ software, using complete linkage and the Pearson correlation coefficient as a measure of similarity.

For each candidate driver and FOXL2, two sets of transcriptomic neighbors were defined by a statistically significant correlation of expression in all tumors $(\mathrm{R}>=0.63$ or $\mathrm{R}<=-0.63)$. These gene sets were analyzed using the Enrichr tool (http://amp.pharm.mssm.edu/Enrichr [10]). The extensive sharing of transcriptomic neighbors between the candidate drivers or FOXL2 was displayed using Cytoscape 3.0.1 software, keeping only the strongly correlated transcriptional neighbors $(R>=0,90)$ for clarity. The network was built using the "prefuse force directed" algorithm with EdgeBetweenness criteria, then manually edited for clarity.

\section{Results and discussion}

CGH of ovarian GCTs shows recurrent chromosomal imbalances

To identify DNA copy number changes in GCTs, we performed a CGH analysis of 10 tumor genomic DNA samples, using microarrays. All the tumors bear the FOXL2 somatic mutation C134W. Four tumors (H1, H8, $\mathrm{H} 28$ and H30) did not display any large-scale genome alterations. However, there was no obvious correlation between the absence of imbalances and tumor stage, size or age of occurrence. On the other extreme, the most altered tumor was $\mathrm{H} 4$, which is not surprising, owing to the fact that it is a recurrence (Additional file 1: Table S1a and S1b).

The detected large-scale imbalances were either recurrent or appeared only once in our samples. Whole-chromosome alterations involved trisomies $8(1 / 10)$ and $14(2 / 10)$, and monosomies 16 (1/10), $21(2 / 10)$ and $22(3 / 10)$. Other long-range changes included duplication of $1 \mathrm{p} 11.1$-qter (H4), and deletions of 1p11.1-p22.1 (H33), 12q13.11q13.13 (H4), 13q13.3-q32.1 (H4), 16p11.2-qter (H4). Our analysis combined with a review of the literature ([11-14]) compiles the data of 94 adult-type GCTs (Figure 1 and Additional file 1: Table S1c). 64 of them presented largescale alterations. This compilation shows the existence of highly recurrent chromosomal alterations, such as supernumerary chromosomes 8, 9, 12 and especially chromosome $14(n=25 / 64$, for the latter) and partial or complete loss of chromosomes 1p, 13q, 16, 21 and particularly 22 $(n=34 / 64$, for the latter). The compiled data also show the co-occurrence of chromosomal alterations, i.e. $-1 \mathrm{p} /-22$ $(\mathrm{n}=5) ;+7 /-16 \mathrm{q}(\mathrm{n}=5) ;+12 /-22(\mathrm{n}=6) ;-13 \mathrm{q} /-22(\mathrm{n}=4) ;$ $+14 /-22(\mathrm{n}=18)$. However, only the $+14 /-22$ and the $+7 /$ $16 \mathrm{q}$ associations were non-random $(\mathrm{p}=0,02$ and $\mathrm{p}=$ 0,001, respectively, according to a two-tailed Fisher's exact test). This suggests that the co-occurrence of $+14 /-22$ and $+7 /-16 q$ imbalances should confer a selective advantage, whose molecular basis remains to be elucidated.

Concerning the FOXL2 locus, all tumors have kept the two alleles, although in two cases the DNA sequence displayed only the presence of the mutated version (data not shown). This can be due to either a second mutational hit or a gene conversion event that provides a selective advantage over heterozygous cells, as previously noted [14].

\section{Large-scale genomic alterations and their transcriptomic translation}

Next, we focused on the genes involved in the altered chromosomal segments and compiled their expression levels. Here, two transcriptomic hybridizations for the same tumor were combined and the average expression level for each gene was computed. Figure $2 \mathrm{a}$ shows that gene expression levels averaged over $\mathrm{Mb}$-sized windows closely reflected the underlying chromosomal imbalances, as detected by CGH.

To further explore the influence of DNA copy number on gene expression, we compared the average expression of genes located in altered segments with that of genes located outside. For example, the copy number increased from 1.01 for the non-amplified segment of chromosome 1 in tumor $\mathrm{H} 4$ to 1.35 for the amplified region (Figure 2b). Consistently, the normalized gene expression averaged over the non-duplicated segment was 0.99 versus 1.21 for the duplicated region. A similar concordance was observed for other amplifications and deletions (Figure $2 \mathrm{~b}$ and data not shown). Although there was a correlation between DNA amounts and mRNA levels, the degree of gene up- or down-regulation was always slightly lower. Although this effect might be due, in some cases, to contamination of tumor RNA with the transcriptome of neighboring normal cells, this explanation cannot apply to all samples. Thus, one is tempted to argue that some degree of expression compensation to chromosome dosage changes is taking place. Indeed, buffering of gene expression in response to genomic alterations have been reported in Drosophila harboring chromosomal imbalances [15-17], for human trisomy 21 [18] and for genes included in Copy-Number Variants (CNVs) [19].

\section{Identification of putative drivers: recurrently broken, amplified/duplicated or deleted genes}

To further exploit our CGH and transcriptomic data, we focused on small-scale rearrangements that might help 


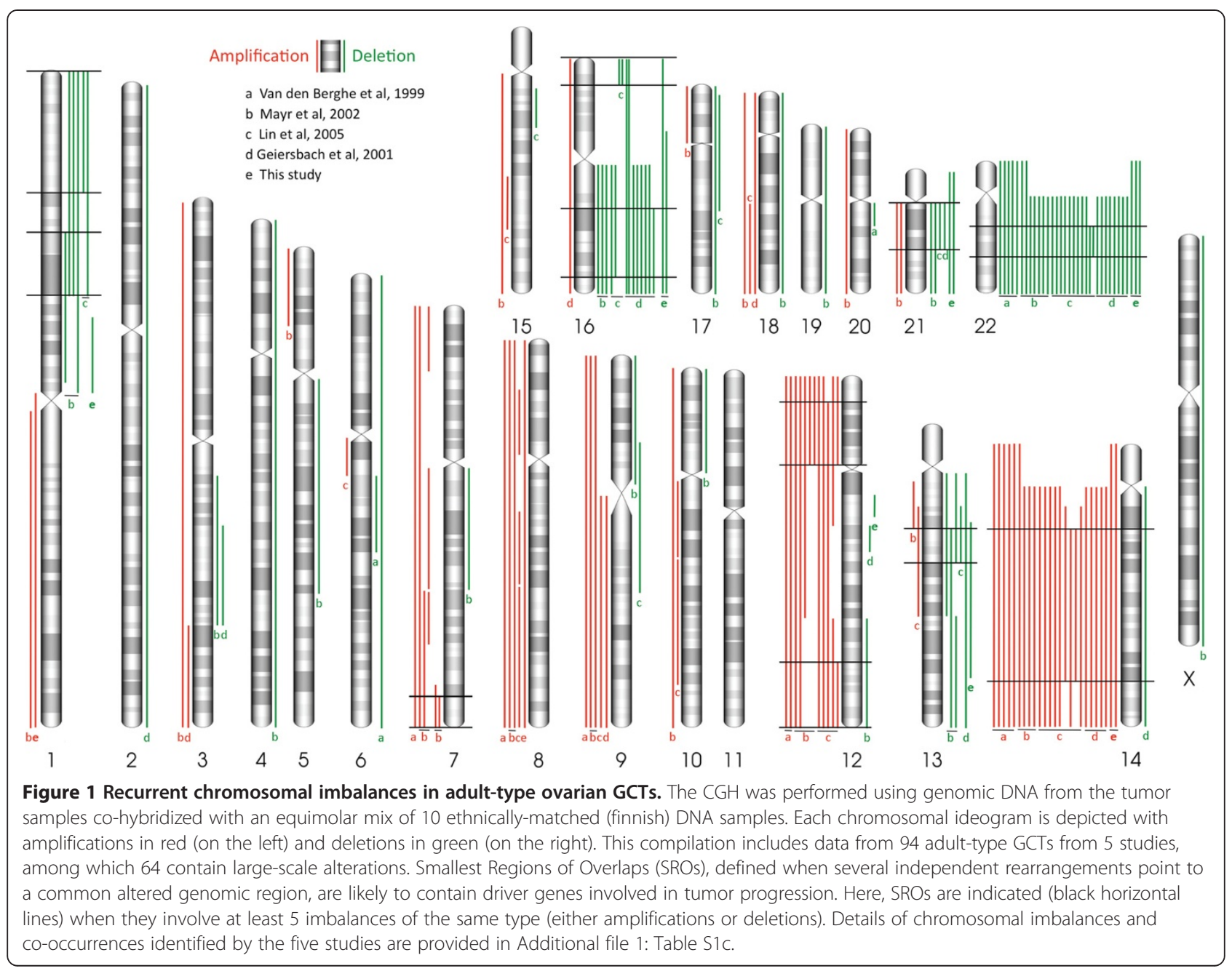

us pinpoint candidate genes whose duplication, deletion or breakage might be involved in tumorigenesis. First, we aimed at identifying amplified or deleted candidate drivers by combining $\mathrm{GCH}$ and transcriptomics. For this purpose, we generated a list of amplified or deleted CGH probes, whose log-ratio corresponded to at least $50 \%$ of the cells harboring a heterozygous duplication or deletion, in at least two tumors. Then, we computed the correlation coefficient (R) over all tumors between the CGH values and the mRNA expression values for the genes whose boundaries mapped at less than $25 \mathrm{~kb}$ from a copynumber-altered probe. This correlation filter was essential because a local genomic alteration does not necessarily imply a transcriptomic change. Thus, a meaningful driver, mapping to an amplified/deleted region, should display a reasonable correlation between copy number and mRNA expression. We set the threshold for statistical significance of Pearson's correlation coefficient R to 0.63 , which is the standard cut-off for ten samples. Genomic regions involved in large-scale imbalances such as trisomies or monosomies were analyzed separately by removing data from trisomic or monosomic tumors. For these regions, the threshold for $\mathrm{R}$ was adjusted to 0.67 or 0.71 in cases when 1 or 2 samples were removed. After excluding genes located within CNVs, we obtained a list of 48 candidates. After manual verification, we retained 13 amplified and 7 deleted genes fully located within the imbalances. Tumors harbored alterations ranging from 2 to $9 / 13$ amplifications and from 1 to $7 / 7$ deletions (Additional file 2: Table S2a).

A literature search shows the known or plausible implication in tumorigenesis for most of these 20 candidates (Table 1). AKT1, encoding a proto-oncogenic kinase, was the most frequently amplified gene (6/10 tumors). AKT1 amplifications have been described in various types of cancer [20-22]. The second most frequently amplified gene (5/10 tumors) encodes the nuclear receptor NR1D1, a survival factor in a subset of breast cancers. Its driver effect might rely on its antiapoptotic activity [23] or on its known upregulation of genes involved in an abnormal 


\section{a}
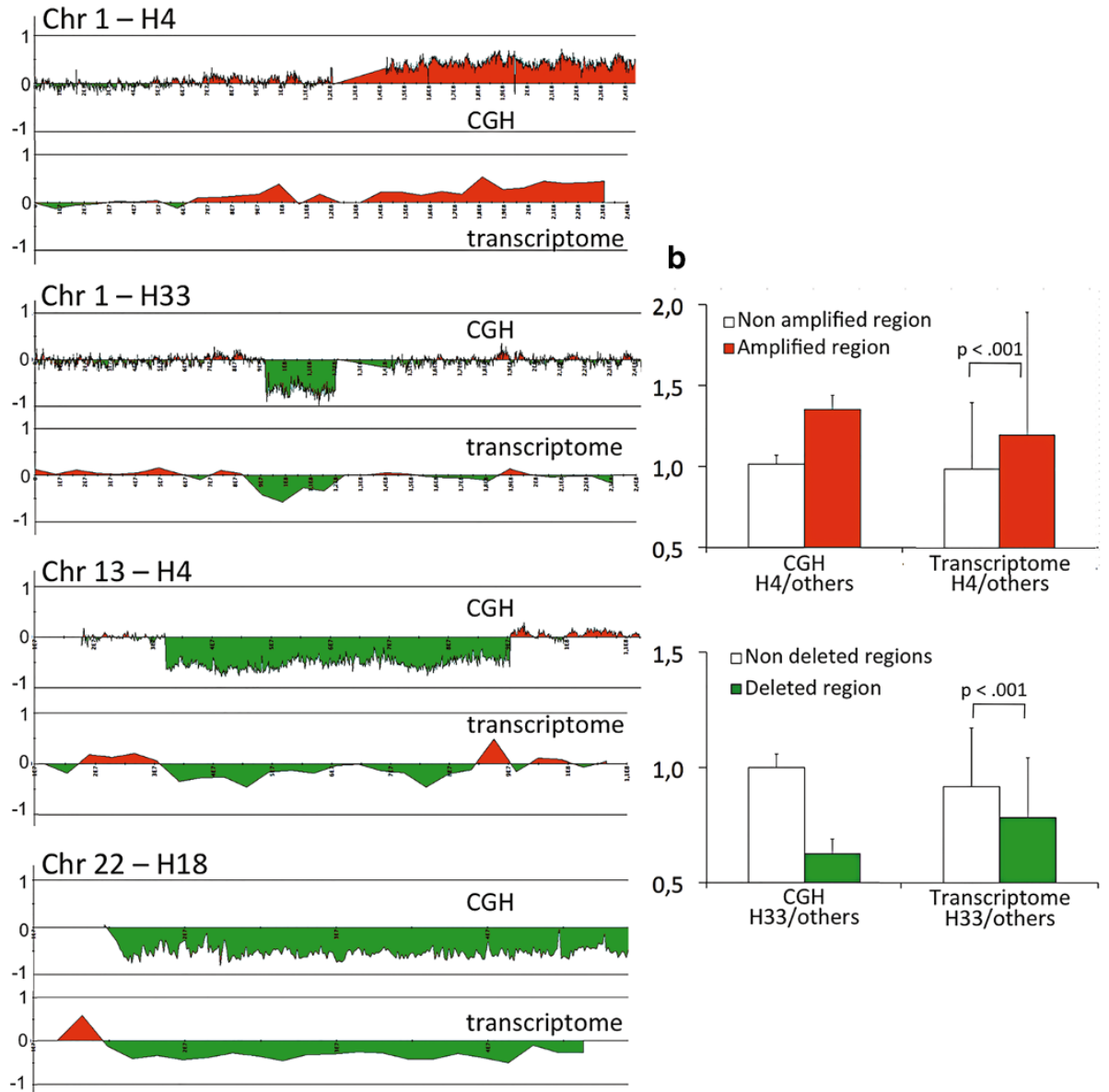

Figure 2 Transcriptomic effects of large-scale genomic rearrangements in adult-type GCTs. a. The CGH data (ratios tumor/reference) are displayed as log2 values averaged for sliding windows of $130 \mathrm{~kb}$ over the relevant chromosomes. For the transcriptomic data, we first computed the average expression levels for each gene (data from two transcriptomic hybridizations). Then we normalized gene expression as described in Methods. Normalized expression values were averaged over 30 windows (of the same size) per chromosome. Notice the close "correlation" between the chromosome copy-number and the expression levels of the genes involved in the imbalances. b. Comparison of the mean CGH values (ratios tumor/ reference, in the natural scale) for the amplified $\mathrm{Chr} 1 \mathrm{q}$ in $\mathrm{H} 4$ or the deleted segment of $\mathrm{Chr} 1$ in $\mathrm{H} 33$ with respect to the rest (non imbalanced) of the chromosome. For the transcriptome, the means of the normalized expression levels for genes located in altered segments (according to CGH) were significantly different from the means for genes located outside on the same chromosome (using both a t-test and a Mann-Whitney non-parametric test).

aerobic glycolysis typical of cancer [24]. $M M A B$, found amplified in 4 out of 10 tumors, encodes the enzyme catalyzing the final step for conversion of vitamin $\mathrm{B}(12)$ into adenosylcobalamin. Interestingly, chemical derivatives of adenosylcobalamin are used to image breast, lung, colon, thyroid, and sarcomatous malignancies [25]. TSPAN32, amplified in 3/10 tumors, encodes a member of the tetraspanin superfamily, and is known to regulate cell proliferation. Along similar lines, another candidate encodes TENC1 (amplified in 2/10 tumors), known to stimulate PI3K/Akt signaling. Furthermore, TENC1 knock-down decreases cell proliferation and its overexpression is associated with aggressive hepatocellular carcinoma [26,27]. This points to a deregulation of the PI3K/AKT pathway in GCTs, that would participate to tumorigenesis $[28,29]$. Another amplified candidate driver (3/10 tumors) encodes RANBP1, a cytoplasmic component of the nuclear pore complex. RANBP1 ensures cargo release from CRM1 upon export of specific mRNAs depending on the oncogenic factor eIF4E [30]. It is worth noting that these last two genes, along with 4 other amplified candidates, were found deleted in one tumor, $\mathrm{H} 4$, which was the only recurrence included in our samples.

Among the recurrently deleted genes, HSPA4, deleted in 3 of the tumors, encodes a chaperone of the HSP110 family, predominantly expressed in the ovary [31]. Interestingly, HSPA4 is known to regulate cell migration, both positively and negatively $[32,33]$. The second gene 
Table 1 Candidate driver genes identified as amplified or deleted in OGCTs, with correlated expression

\begin{tabular}{|c|c|c|c|c|c|}
\hline \multirow[t]{2}{*}{ Chr } & \multirow[t]{2}{*}{ Gene } & \multicolumn{2}{|c|}{ \# of tumors with } & \multirow[t]{2}{*}{ Function \& Implication in cancer if known } & \multirow[t]{2}{*}{ Ref } \\
\hline & & Amp & Del & & \\
\hline 14 & AKT1 & 6 & 0 & Known oncogenic kinase, core of one of the most frequently activated survival pathways in human cance. & [50] \\
\hline 17 & NR1D1 & 5 & 0 & $\begin{array}{l}\text { Ligand-sensitive transcription factor, regulates the expression of core clock proteins; required for survival } \\
\text { and proliferation of breast cancers }\end{array}$ & [24] \\
\hline 12 & $M M A B$ & 4 & 0 & $\begin{array}{l}\text { catalyzes the final step for conversion of vitamin } \mathrm{B}(12) \text { into adenosylcobalamin. Derivatives of the latter } \\
\text { are used to image breast, lung, colon, thyroid, and sarcomatous malignancies. }\end{array}$ & [25] \\
\hline 11 & TSPAN32 & 3 & 0 & $\begin{array}{l}\text { Membrane protein, regulates T cell proliferative responses. Tetraspanins are implicated in various steps } \\
\text { of tumorigenesis. }\end{array}$ & [51] \\
\hline 11 & CTSW & 2 & 0 & cysteine proteinase up-regulated in Large granular lymphocyte leukemia & [52] \\
\hline 12 & $D G K A$ & 2 & 0 & $\begin{array}{l}\text { converts DAG into PA, a second messenger activating multiple signaling pathways implicated in } \\
\text { tumorigenesis (i.e. mTOR signaling) }\end{array}$ & [53] \\
\hline 22 & RANBP1 & 3 & $1^{*}$ & $\begin{array}{l}\text { Soluble component of the nuclear pore complex. Oncogenic overexpression of elF4E induces } \\
\text { overexpression of RANBP1 }\end{array}$ & [30] \\
\hline 22 & TRMT2A & 3 & $1^{*}$ & $\begin{array}{l}\text { cell-cycle regulated protein, one of the } 5 \text { immunohistochemical markers in the Mammostrat test used } \\
\text { to stratify breast cancers }\end{array}$ & {$[54]$} \\
\hline 12 & TENC1 & 2 & $1^{*}$ & $\begin{array}{l}\text { Promotes PI3K/Akt signaling, KD }=>\text { decreased proliferation. High expression associated with aggressive } \\
\text { hepatocellular carcinoma }\end{array}$ & {$[26]$} \\
\hline 16 & PIEZO1 & 2 & $1^{*}$ & $\begin{array}{l}\text { transmembrane protein involved in mechanotransduction. Mediates integrin activation by recruiting } \\
\text { R-Ras to the ER, modulating cell adhesion }\end{array}$ & [55] \\
\hline 12 & SPRYD3 & 2 & $1^{*}$ & SPRY domain containing 3. Not studied & na \\
\hline 22 & C22orf26 & 3 & $2^{*}$ & Not studied - now named PRR34, proline rich 34 & na \\
\hline 22 & FAM19A5 & 3 & 2 & $\begin{array}{l}\text { postulated to function as brain-specific chemokines or neurokines, acting as regulators of immune and } \\
\text { nervous cells. }\end{array}$ & [56] \\
\hline 1 & $N V L$ & $1^{*}$ & 3 & $\begin{array}{l}\text { AAA-ATPase, hTERT binding, essential for telomerase assembly. A nucleolar isoform is a component of } \\
\text { pre-ribosomal particles }\end{array}$ & [36] \\
\hline 19 & C19orf18 & 0 & 2 & not studied. Identified as significantly binding to oligomeric $\beta$-amyloid & [57] \\
\hline 14 & FAM177A1 & 0 & 2 & $\begin{array}{l}\text { Unknown function. Down-regulated by microRNA124 during neurogenesis. Identified as a target of } \\
\text { the E3 ubiquitin-ligase FANCA. }\end{array}$ & \\
\hline 12 & LIMA1 & 0 & 2 & $\begin{array}{l}\text { Inhibits actin depolymerization and cross-links filaments in bundles. Putative suppressor of } \\
\text { epithelial-mesenchymal transition and metastasis }\end{array}$ & \\
\hline 17 & $T A D A 2 A$ & 0 & 2 & $\begin{array}{l}\text { transcriptional activator adaptor, in the PCAF and ATAC histone acetylase complexes, mediates DNA } \\
\text { damage-induced apoptosis and G1/S arrest }\end{array}$ & \\
\hline 5 & HSPA4 & 0 & 3 & $\begin{array}{l}\text { Heat shock chaperone of the HSP110 family. Regulates cell proliferation and G1/S progression by } \\
\text { releasing transcription factor ZONAB from tight junction sequestration }\end{array}$ & {$[59$} \\
\hline 15 & RTF1 & 0 & 3 & $\begin{array}{l}\text { part of the Paf1/RNA polymerase II complex, key regulator of transcription-related processes and } \\
\text { cell-cycle progression }\end{array}$ & \\
\hline
\end{tabular}

*These genes were found altered in the opposite way in the tumor $\mathrm{H} 4$, the only recurrent tumor in our cohort.

deleted in 3/10 tumors is RTF1, encodes a member of the Paf1 complex, which is a key regulator of RNA polymerase II transcriptional activity and of cell-cycle progression. RTF1 is critical for histone and chromatin modifications and telomeric silencing [34,35]. Another link with telomere maintenance is $N V L$, also found deleted in 3 tumors. NVL encodes an AAA-ATPase essential for hTERT binding and telomerase assembly [36]. In addition, a nucleolar isoform of NVL participates in ribosome biosynthesis [37]. LIMA1 (a.k.a. EPLIN, Epithelial protein lost in neoplasm), deleted in 2/10 OGCTs, encodes a metastasis suppressor, frequently lost in cancer cells $[38,39]$. Consistently, it acts as a negative regulator of epithelial-mesenchymal transition and invasiveness [40] and its expression is inversely correlated with the aggressiveness of breast cancer [41]. Another interesting
Table 2 Genes identified as broken in OGCTs

\begin{tabular}{|c|c|c|c|}
\hline Chr & Gene & Function \& Implication in cancer if known & Ref \\
\hline 8 & C8orf34 & $\begin{array}{l}\text { CAMP-dependent protein kinase regulator. } \\
\text { Associated with irinotecan-related toxicities } \\
\text { in patients with non-small-cell lung cancer. }\end{array}$ & {$[60]$} \\
\hline 18 & CELF4 & $\begin{array}{l}\text { CELF/BRUNOL protein, alternative splicing factor. } \\
\text { When lost, independent prognostic indicator in } \\
\text { colorectal cancer. }\end{array}$ & [61] \\
\hline 14 & NPAS3 & $\begin{array}{l}\text { Basic helix-loop-helix and PAS domain-containing } \\
\text { transcription factor, tumor suppressor in astrocytomas }\end{array}$ & {$[62]$} \\
\hline 15 & SPG11 & $\begin{array}{l}\text { Potential transmembrane protein phosphorylated } \\
\text { upon DNA damage. Mutated in recessive } \\
\text { hereditary spastic paraplegia. }\end{array}$ & [63] \\
\hline 21 & RUNX1 & $\begin{array}{l}\text { CBF transcription factor subunit. Tumor suppressor, } \\
\text { with oncogenic fusions in leukemias and mutations } \\
\text { in breast cancers. }\end{array}$ & [64] \\
\hline
\end{tabular}


deleted gene, TADA2A, encodes an adaptor subunit of the PCAF and ATAC histone acetylase complexes. TADA2Acontaining PCAF complex is essential for DNA-damageinduced acetylation of $\mathrm{p} 53$, necessary to promote cell cycle arrest and cell survival after DNA damage [42,43]. Moreover, TADA2A overexpression is pro-apoptotic in response to DNA damage [44]. Thus, its deletion in GCTs should provide resistance to apoptosis [45]. FOXO factors

\section{a}

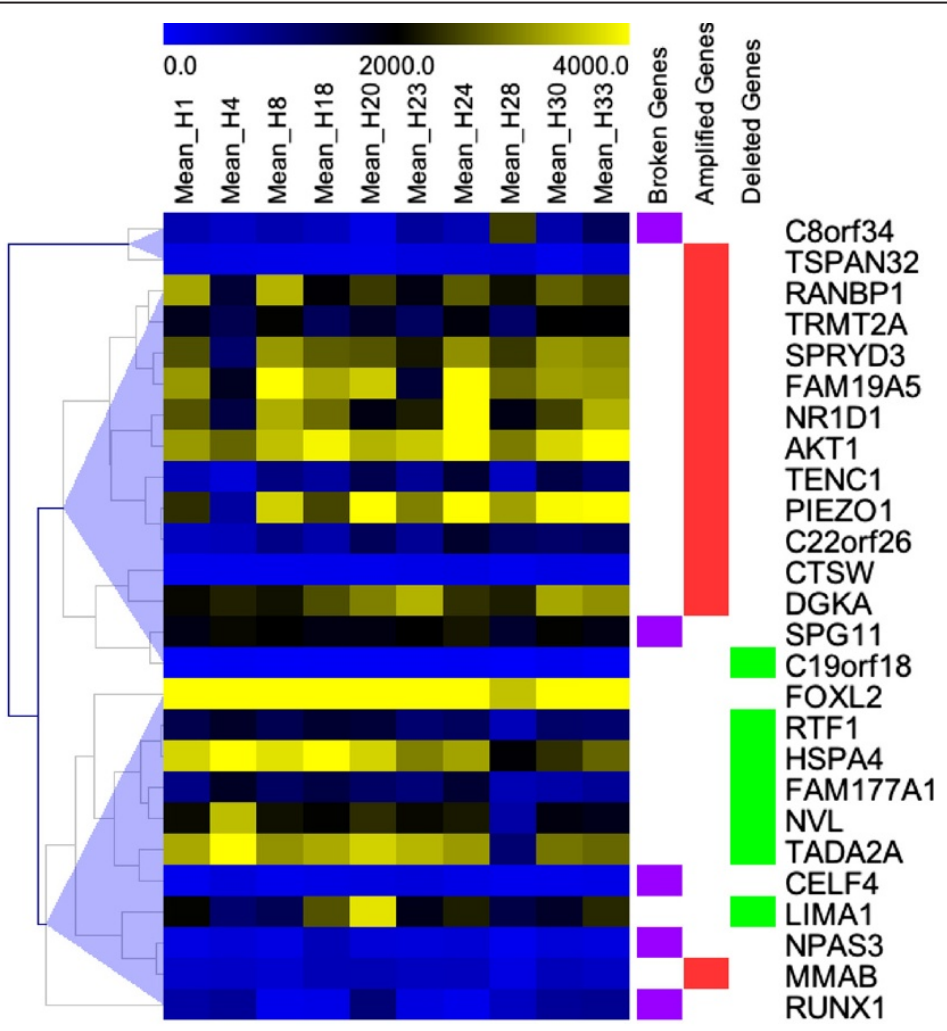

b

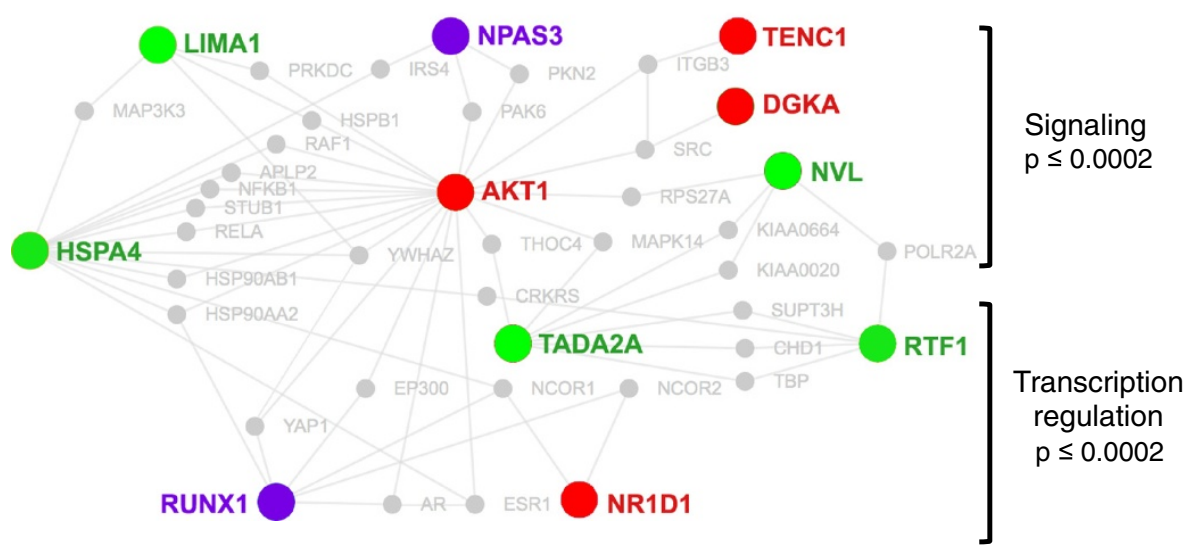

Figure 3 Functional relationships between broken, amplified and deleted candidates drivers in adult-type GCTs. a. Hierarchical clustering of the expression levels of the 5 broken genes (purple), 13 amplified (red), 7 deleted candidate drivers (green), and FOXL2 in the 10 GCTs (see details in Methods). The clustering defines three groups of genes. The first group contains 5 deleted putative drivers together with the majority of broken genes, amplified MMAB and FOXL2. The second group includes almost all amplified genes, and one broken and 1 deleted genes. Amplified TSPAN32 (anti-correlated to other amplified genes) defines a separate group along with the remaining broken gene. b. Physical interaction network involving the proteins encoded by broken, amplified or deleted candidate drivers and common binding partners. Known interactions were retrieved automatically by DAPPLE v2.0 (http://www.broadinstitute.org/mpg/dapple/dappleTMP.php, see [48]), using default parameters. The network was manually reorganized to highlight the expected hub position of AKT1 and the partition of identified binding partners in signaling and transcription regulation. Gene set enrichment analysis was performed with Enrichr, for the 43 genes depicted in the network (the displayed p-values are Bonferroni-corrected). 
are known to be acetylated by PCAF upon stress to promote cycle arrest and DNA damage repair, or apoptosis. We have previously shown that FOXL2 is acetylated [46] and that it upregulates stress-response genes and induces cell-cycle slow-down [8]. A hyperlinked gene list with more complete information is provided in Additional file 2: Table S2a and S2b.

Next, we identified genes recurrently broken in the tumor samples. They were pinpointed by the existence, in at least 2 tumors, of one or several closely mapping breakpoints defined by amplifications/deletions upstream and downstream, within the relevant gene. A literature search for the 5 genes identified as broken showed that 4 of them are clearly involved in cancer (Table 2, more details are provided in Additional file 2: Table S2a and S2b). In particular, NPAS3 and RUNX1 are known tumor suppressors and CELF4 is known to be frequently deleted in cancer. Broken genes might be fusion partners, as described for RUNX1 in leukaemia [47], although we have no direct evidence for this.

\section{The candidate drivers are expressionally clustered and share transcriptomic neighbors}

To explore possible functional links among the candidate drivers, we performed a standard hierarchical clustering of the expression levels of the $20 \mathrm{amplified} /$ deleted candidate drivers, the 5 broken genes and FOXL2 (Figure 3A). This analysis defined three main groups: group 1 contained 6/7 deleted genes (i.e. NVL, RTF1, TADA2A, HSPA4, FAM177A1, LIMA1), 3/5 broken genes (which is coherent with a loss of function), 1 amplified gene $(M M A B)$ and FOXL2 itself; group 2 involved 11/13 amplified genes (including AKT1), 1 broken one and 1 deleted gene (C19orf18); and group 3 involved 1 broken gene (C8orf34) along with amplified TSPAN32. Functional links between these genes are supported by their interactions with common partners (Figure 3b), as detected by Dapple2 for $11 / 25$ genes [48].

To further explore the implication of amplified/deleted candidate drivers in processes altered in cancer, we determined for each of them a list of positive and negative

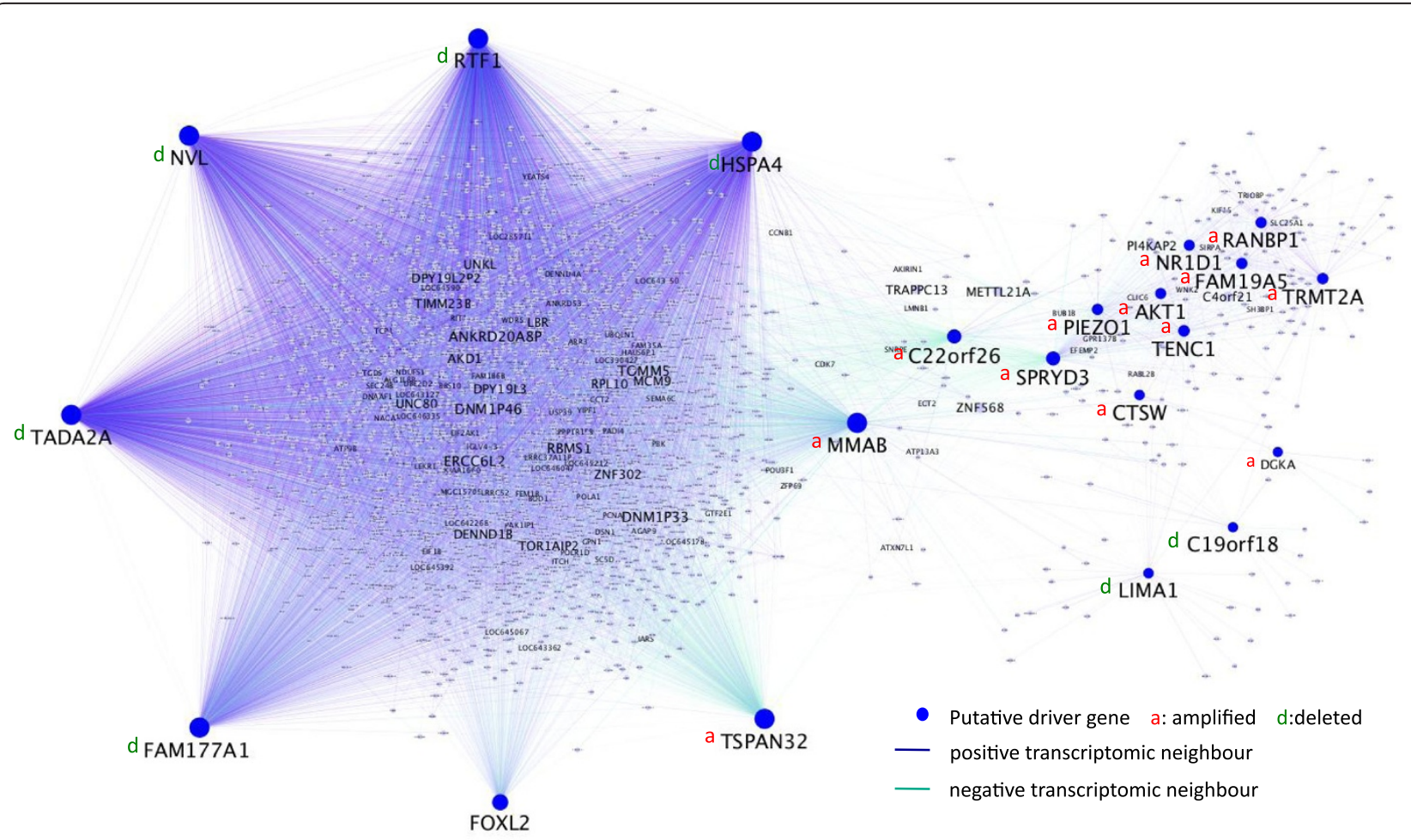

Figure 4 Sharing of transcriptional neighbors among amplified/deleted genes. The 20 putative drivers and FOXL2 (blue nodes) are depicted within a network with strongly correlated transcriptional neighbors $(R>=0,90$ ), either positively (blue edges) or negatively (green edges). The diameter of the nodes reflects the number of neighbors. Amplified genes are labeled with a red $a$, and deleted ones with a green $d$. A high-resolution zoomable network is provided in Additional file 3: Figure S1. Notice that extensive sharing of transcriptomic neighbors parallels the same groups of candidate drivers than the expressional correlation in Figure 3a. Five of the deleted genes in the first group in Figure 3a share many positive and negative neighbors, and those neighbors are mainly negatively connected to the only amplified gene of this group, MMAB. The amplified genes of the second cluster (from Figure 2a) are grouped in a distinct sub-network with a connection to the dense sub-network of deleted candidate drivers restricted to C22orf26 and SPRYD3. Amplified TSPAN32 has a peculiar position, as it is connected only to neighbors of the first dense sub-network. Large grey nodes depict transcriptomic neighbors that are connected to a large portion of the candidate drivers (i.e. POU3F1, MCM9, RPL10, POLR1D, PCNA, POLA1, PIAKAP2). 
"transcriptomic neighbors" (i.e. genes whose expression levels displayed $\mathrm{R}>=0.63$ or $\mathrm{R}=<-0.63$ with the expression of the relevant driver over the tumor samples). Such transcriptomic (positive and negative) neighbors are more likely to be functionally linked to the drivers than random genes. A gene ontology analysis using EnrichR [10] showed that the negative transcriptomic neighbors of amplified drivers and the positive neighbors of deleted drivers were enriched in keywords involving cell cycle, suggesting a coherent effect of these two types of alterations. Other enriched keywords pointed to different types of cancer, DNA damage response, DNA repair and regulation of ubiquitinylation during mitosis (Additional file 2: Table S2c). Interestingly, all the candidate drivers shared a statistically significant proportion of transcriptomic neighbors. The network of Figure 4 (and Additional file 3: Figure S1) shows several interesting points: i) 5 of the deleted genes (NVL, RTF1, TADA2A, HSPA4, $F A M 177 A 1)$ included in group 1 share a highly significant number of transcriptomic neighbors and form a dense sub-network; ii) four amplified genes, MMAB, SPRYD3, C22orf26 and TSPAN32 are anti-correlated with a large number of positive neighbors of these 5 deleted putative drivers; iii) the amplified genes included in the expression group 2 also share transcriptomic neighbors and iv) FOXL2 is heavily connected to the deleted putative drivers, which suggests an interplay between the C134W mutation and the genomic alterations (especially the deletions) that we have detected.

\section{Conclusions}

In conclusion, our analysis identifies candidate co-driver genes, whose various alterations could contribute to GCT pathogenesis besides the FOXL2 somatic mutation. This is strengthened by their high degree of expressional interconnection, which suggests the existence of functional interactions among them, and by their known or suggested implication in cancer and related processes. However, we are aware that, given the small sample size for which $\mathrm{CGH}$ and transcriptomic data were available, this genomic exploration only provides leads for functional analyses to formally demonstrate the implication of the candidate drivers in GC tumorigenesis.

\section{Additional files}

Additional file 1: Table S1. Tab a - Rainbow overview of genomic alterations in 10 adult ovarian GCTs. The CGH data is displayed as averaged values for sliding $130 \mathrm{~kb}$ windows, in log2 scale. In that scale, equivalent amount of genomic DNA for the tumor sample and the control DNA is displayed as values around the baseline at 0 , segments below 0 indicate deletions, and segment above 0 indicate amplifications. Each chromosome is depicted with a different color. Tab b - Details of large-scale genomic alterations detected by CGH in the $10 \mathrm{GCTs}$. The clinical details were previously published in [49]. The recurrent alterations are indicated in bold. Tab c - Details of large-scale genomic alterations detected in ovarian GCTs by five different studies. The alterations are listed by chromosome. Clinical details are indicated when available. For each tumor, the alteration of the chromosome is written in black, other rearrangements present in the same tumors are indicated in grey and in parenthesis. Note that for acrocentric chromosomes, an alteration of the $\mathrm{q}$ arm is indicative of the amplification or loss of the whole chromosome (i.e. trisomy or monosomy). The last two columns highlight the associated recurrent alterations, and indicate whether these are statistically overrepresented (two-tailed p. Fisher's exact test on a contingency table).

Additional file 2: Table S2. Tab a - List of broken, amplified and deleted candidate driver genes. This table is a more complete, detailed and hyperlinked version of Tables 1 and 2. Genes altered by breakpoint were identified by upstream and downstream rearranged CGH status in at least 2 tumors. The breakpoint must be within the gene in all the tumors, and not included in frequent CNVs detected in healthy control samples. Candidate driver genes were identified as presenting an expression significantly correlated (correlation coefficient $>=0.63$ ) with the CGH status, in at least 2 tumors, without being included in CNVs detected in healthy control samples. CNVs were verified in DGV database, that contains the genomic alterations involving segments of DNA that are larger than $>50 \mathrm{bp}$ identified in healthy control samples (DGV update: 31/05/2013). Tab b - Details of the genomic alterations leading to the identification of broken genes in ovarian GCTs. For each breakpoint are indicated: the tumors displaying an alteration, the genomic coordinates of the alterations, the name of the broken gene, bibliographic data centered on cancer-related processes, and the screenshot of the display in $\mathrm{MeV}$ software. Bright red and green regions are genomic segments respectively amplified or deleted in more than $50 \%$ of the cells in the tumor samples. Tab c - Keywords enrichment of transcriptomic neighbors lists for the 20 putative amplified/deleted drivers. For each candidate driver, the sets of transcriptomic neighbors with a statistically significant correlation of expression $(R>=0.63$ or $R<=-0.63$ ) were tested using Enrichr. The keywords in KEGG pathways and in Gene Ontology Biological Process (GO-BP) are given for positive and negative neighbors, when they reached significant enrichment after correction of the $p$-value by the Bonferroni method. Cancer-related keywords are highlighted in red.

Additional file 3: Figure S1. High-resolution image of the network between the 20 candidate drivers and their highly-correlated transcriptomic neighbors. Blue nodes: 20 candidate drivers and FOXL2. Amplified genes are labeled with a red a, and deleted ones with a green $\mathrm{d}$. Grey nodes: transcriptomic neighbors with expression correlated with a correlation coefficient $>=0.90$ to at least one of the candidate drivers or FOXL2. The sizes of the node and of the node label are proportional to the number of edges. Some transcriptomic neighbors are connected to a large portion of the candidate drivers (large grey nodes). Purple edge: positive correlation between the candidate driver (or FOXL2) and its transcriptomic neighbor. Green edge: negative correlation between the candidate driver (or FOXL2) and its transcriptomic neighbor. The edges are rendered semi-transparent in order to keep the gene names visible. The picture is zoomable to see individual gene names. The Cytoscape session file is available upon request.

\section{Competing interests}

The authors declare that they have no competing interest.

\section{Authors' contributions}

SC: designed study, analyzed samples and drafted MS. MA: designed study, analyzed samples and drafted MS. ALT: performed molecular genetic studies. LUK: provided samples, analyzed data and drafted MS. DM: performed bioinformatics operations and statistical analyses. RB: provided samples, analyzed data and drafted MS. RAV: designed study, analyzed samples and drafted MS. All authors read and approved the final manuscript.

\section{Acknowledgements}

We gratefully acknowledge financial support from the Centre National de la Recherche Scientifique, La Ligue Nationale contre le Cancer (Comité de Paris), I'Université Paris Diderot-Paris7, I'Institut Universitaire de France, the Academy of Finland, and Helsinki University Central Hospital Research Funds. 
We thank A-E. Lehesjoki for providing the Finnish control DNA pool, and M. Heikinheimo for support on the GCT research program in Helsinki.

\section{Author details}

${ }^{1}$ Institut Jacques Monod, Paris, France. ${ }^{2}$ Université Paris Diderot/Paris, Paris, France. ${ }^{3}$ Department of Obstetrics and Gynecology, University of Helsinki and Helsinki University Central Hospital, Helsinki, Finland. ${ }^{4}$ Children's Hospital, University of Helsinki and Helsinki University Central Hospital, Helsinki, Finland. ${ }^{5}$ Department of pathology, University of Helsinki, and HUSLAB, Helsinki University Central Hospital, Helsinki, Finland. ${ }^{6}$ Université Paris-Diderot \& Institut Jacques Monod, CNRS-UMR 7592, Bâtiment Buffon, 15 Rue Hélène Brion, Paris, Cedex 13, France.

\section{Received: 21 August 2014 Accepted: 27 March 2015}

\section{Published online: 10 April 2015}

\section{References}

1. Pectasides D, Pectasides E, Psyrri A. Granulosa cell tumor of the ovary. Cancer Treat Rev. 2008;34:1-12.

2. Schumer ST, Cannistra SA. Granulosa cell tumor of the ovary. J Clin Oncol. 2003;21:1180-9.

3. East N, Alobaid A, Goffin F, Ouallouche K, Gauthier P. Granulosa cell tumour: a recurrence 40 years after initial diagnosis. J Obstet Gynaecol Can. 2005;27:363-4.

4. Shah SP, Köbel M, Senz J, Morin RD, Clarke BA, Wiegand KC, et al. Mutation of FOXL2 in granulosa-cell tumors of the ovary. N Engl J Med. 2009;360:2719-29.

5. Benayoun BA, Caburet S, Dipietromaria A, Georges A, D'Haene B, Pandaranayaka PJE, et al. Functional exploration of the adult ovarian granulosa cell tumor-associated somatic FOXL2 mutation p.Cys134Trp (c.402C > G). PLoS One. 2010;5:e8789.

6. Rosario R, Araki H, Print CG, Shelling AN. The transcriptional targets of mutant FOXL2 in granulosa cell tumours. PLoS One. 2012;7:e46270.

7. Kim J-H, Yoon S, Park M, Park H-O, Ko J-J, Lee K, et al. Differential apoptotic activities of wild-type FOXL2 and the adult-type granulosa cell tumor-associated mutant FOXL2 (C134W). Oncogene. 2011;30:1653-63.

8. Benayoun BA, Georges AB, L'Hôte D, Andersson N, Dipietromaria A, Todeschini A- $L$, et al. Transcription factor FOXL2 protects granulosa cells from stress and delays cell cycle: role of its regulation by the SIRT1 deacetylase. Hum Mol Genet. 2011;20:1673-86.

9. Benayoun BA, Anttonen M, L'Hôte D, Bailly-Bechet M, Andersson N, Heikinheimo $M$, et al. Adult ovarian granulosa cell tumor transcriptomics: prevalence of FOXL2 target genes misregulation gives insights into the pathogenic mechanism of the p.Cys134Trp somatic mutation. Oncogene. 2013;32:2739-46.

10. Chen EY, Tan CM, Kou Y, Duan Q, Wang Z, Meirelles GV, et al. Enrichr: interactive and collaborative HTML5 gene list enrichment analysis tool. BMC Bioinformatics. 2013;14:128.

11. Van den Berghe I, Dal Cin P, De Groef K, Michielssen P, Van den Berghe H. Monosomy 22 and trisomy 14 may be early events in the tumorigenesis of adult granulosa cell tumor. Cancer Genet Cytogenet. 1999;112:46-8.

12. Mayr D, Kaltz-Wittmer C, Arbogast S, Amann G, Aust DE, Diebold J. Characteristic pattern of genetic aberrations in ovarian granulosa cell tumors. Mod Pathol. 2002;15:951-7.

13. Lin Y-S, Eng H-L, Jan Y-J, Lee H-S, Ho WL, Liou C-P, et al. Molecular cytogenetics of ovarian granulosa cell tumors by comparative genomic hybridization. Gynecol Oncol. 2005;97:68-73.

14. Geiersbach KB, Jarboe EA, Jahromi MS, Baker CL, Paxton CN, Tripp SR, et al. FOXL2 mutation and large-scale genomic imbalances in adult granulosa cell tumors of the ovary. Cancer Genet. 2011;204:596-602.

15. Gupta V, Parisi M, Sturgill D, Nuttall R, Doctolero M, Dudko OK, et al. Global analysis of X-chromosome dosage compensation. J Biol. 2006;5:3.

16. McAnally AA, Yampolsky LY. Widespread transcriptional autosomal dosage compensation in Drosophila correlates with gene expression level. Genome Biol Evol. 2010;2:44-52.

17. Malone JH, Cho D-Y, Mattiuzzo NR, Artieri CG, Jiang L, Dale RK, et al. Mediation of Drosophila autosomal dosage effects and compensation by network interactions. Genome Biol. 2012;13:r28.

18. Ait Yahya-Graison E, Aubert J, Dauphinot L, Rivals I, Prieur M, Golfier G, et al. Classification of human chromosome 21 gene-expression variations in Down syndrome: impact on disease phenotypes. Am J Hum Genet. 2007;81:475-91.
19. Woodwark C, Bateman A. The characterisation of three types of genes that overlie copy number variable regions. PLoS One. 2011;6:e14814.

20. Banck MS, Kanwar R, Kulkarni AA, Boora GK, Metge F, Kipp BR, et al. The genomic landscape of small intestine neuroendocrine tumors. J Clin Invest. 2013;123:2502-8.

21. De Marco C, Rinaldo N, Bruni P, Malzoni C, Zullo F, Fabiani F, et al. Multiple genetic alterations within the PI3K pathway are responsible for AKT activation in patients with ovarian carcinoma. PLoS One. 2013:8:e55362.

22. Dobashi Y, Kimura M, Matsubara H, Endo S, Inazawa J, Ooi A. Molecular alterations in AKT and its protein activation in human lung carcinomas. Hum Pathol. 2012:43:2229-40.

23. Okazawa S, Furusawa Y, Kariya A, Hassan MA, Arai M, Hayashi R, et al. Inactivation of DNA-dependent protein kinase promotes heat-induced apoptosis independently of heat-shock protein induction in human cancer cell lines. PLoS One. 2013;8:e58325.

24. Kourtidis A, Jain R, Carkner RD, Eifert C, Brosnan MJ, Conklin DS. An RNA interference screen identifies metabolic regulators NR1D1 and PBP as novel survival factors for breast cancer cells with the ERBB2 signature. Cancer Res. 2010;70:1783-92.

25. Collins DA, Hogenkamp HP, O'Connor MK, Naylor S, Benson LM, Hardyman TJ, et al. Biodistribution of radiolabeled adenosylcobalamin in patients diagnosed with various malignancies. Mayo Clin Proc. 2000;75:568-80.

26. Jung AS, Kaushansky A, Macbeath G, Kaushansky K. Tensin2 is a novel mediator in thrombopoietin (TPO)-induced cellular proliferation by promoting Akt signaling. Cell Cycle. 2011;10:1838-44.

27. Yam JWP, Ko FCF, Chan C-Y, Yau T-O, Tung EKK, Leung TH-Y, et al. Tensin2 variant 3 is associated with aggressive tumor behavior in human hepatocellular carcinoma. Hepatology. 2006;44:881-90.

28. Laguë M-N, Paquet M, Fan H-Y, Kaartinen MJ, Chu S, Jamin SP, et al. Synergistic effects of Pten loss and WNT/CTNNB1 signaling pathway activation in ovarian granulosa cell tumor development and progression. Carcinogenesis. 2008;29:2062-72.

29. Bittinger S, Alexiadis M, Fuller PJ. Expression status and mutational analysis of the PTEN and P13K subunit genes in ovarian granulosa cell tumors. Int J Gynecol Cancer. 2009;19:339-42.

30. Culjkovic-Kraljacic B, Baguet A, Volpon L, Amri A, Borden KLB. The oncogene elF4E reprograms the nuclear pore complex to promote mRNA export and oncogenic transformation. Cell Rep. 2012;2:207-15.

31. Kaneko Y, Kimura T, Kishishita M, Noda Y, Fujita J. Cloning of apg-2 encoding a novel member of heat shock protein 110 family. Gene. 1997;189:19-24.

32. Wu C-Y, Lin C-T, Wu M-Z, Wu K-J. Induction of HSPA4 and HSPA14 by NBS1 overexpression contributes to NBS1-induced in vitro metastatic and transformation activity. J Biomed Sci. 2011;18:1.

33. Sakurai T, Kashida H, Hagiwara S, Nishida N, Watanabe T, Fujita J, et al. Heat Shock Protein A4 Controls Cell Migration and Gastric Ulcer Healing. Dig Dis Sci. 2015. Epub ahead of print: 06 Feb 2015.

34. Jaehning JA. The Paf1 complex: platform or player in RNA polymerase II transcription? Biochim Biophys Acta. 2010;1799:379-88.

35. Moniaux N, Nemos C, Deb S, Zhu B, Dornreiter I, Hollingsworth MA, et al. The human RNA polymerase II-associated factor 1 (hPaf1): a new regulator of cell-cycle progression. PLoS One. 2009;4:e7077.

36. Her J, Chung IK. The AAA-ATPase NVL2 is a telomerase component essential for holoenzyme assembly. Biochem Biophys Res Commun. 2012;417:1086-92.

37. Nagahama M, Yamazoe T, Hara Y, Tani K, Tsuji A, Tagaya M. The AAA-ATPase NVL2 is a component of pre-ribosomal particles that interacts with the DExD/ H-box RNA helicase DOB1. Biochem Biophys Res Commun. 2006;346:1075-82.

38. Maul RS, Chang DD. EPLIN, epithelial protein lost in neoplasm. Oncogene. 1999;18:7838-41.

39. Song Y, Maul RS, Gerbin CS, Chang DD. Inhibition of anchorage-independent growth of transformed NIH3T3 cells by epithelial protein lost in neoplasm (EPLIN) requires localization of EPLIN to actin cytoskeleton. Mol Biol Cell. 2002;13:1408-16

40. Zhang S, Wang X, Osunkoya AO, lqbal S, Wang Y, Chen Z, et al. EPLIN downregulation promotes epithelial-mesenchymal transition in prostate cancer cells and correlates with clinical lymph node metastasis. Oncogene. 2011;30:4941-52.

41. Jiang WG, Martin TA, Lewis-Russell JM, Douglas-Jones A, Ye L, Mansel RE. Eplin-alpha expression in human breast cancer, the impact on cellular migration and clinical outcome. Mol Cancer. 2008;7:71. 
42. Liu L, Scolnick DM, Trievel RC, Zhang HB, Marmorstein R, Halazonetis TD, et al. p53 sites acetylated in vitro by PCAF and p300 are acetylated in vivo in response to DNA damage. Mol Cell Biol. 1999;19:1202-9.

43. Knights CD, Catania J, Di Giovanni S, Muratoglu S, Perez R, Swartzbeck A, et al. Distinct p53 acetylation cassettes differentially influence geneexpression patterns and cell fate. J Cell Biol. 2006;173:533-44.

44. Huang J, Zhang L, Liu W, Liao Q, Shi T, Xiao L, et al. CCDC134 interacts with hADA2a and functions as a regulator of hADA2a in acetyltransferase activity, DNA damage-induced apoptosis and cell cycle arrest. Histochem Cell Biol. 2012;138:41-55.

45. Cohen HY, Lavu S, Bitterman KJ, Hekking B, Imahiyerobo TA, Miller C, et al. Acetylation of the $C$ terminus of Ku70 by CBP and PCAF controls Bax-mediated apoptosis. Mol Cell. 2004;13:627-38.

46. Georges A, Benayoun BA, Marongiu M, Dipietromaria A, L'Hôte D, Todeschini A-L, et al. SUMOylation of the Forkhead transcription factor FOXL2 promotes its stabilization/activation through transient recruitment to PML bodies. PLoS One. 2011;6:e25463.

47. Guastadisegni MC, Lonoce A, Impera L, Di Terlizzi F, Fugazza G, Aliano S, et al. CBFA2T2 and C20orf112: two novel fusion partners of RUNX1 in acute myeloid leukemia. Leukemia. 2010;24:1516-9.

48. Rossin EJ, Lage K, Raychaudhuri S, Xavier RJ, Tatar D, Benita Y, et al. Proteins encoded in genomic regions associated with immune-mediated disease physically interact and suggest underlying biology. PLoS Genet. 2011;7:e1001273.

49. Jamieson S, Butzow R, Andersson N, Alexiadis M, Unkila-Kallio L, Heikinheimo M, et al. The FOXL2 C134W mutation is characteristic of adult granulosa cell tumors of the ovary. Mod Pathol. 2010;23:1477-85.

50. Hers I, Vincent EE, Tavaré JM. Akt signalling in health and disease. Cell Signal. 2011;23:1515-27.

51. Gartlan KH, Belz GT, Tarrant JM, Minigo G, Katsara M, Sheng K-C, et al. A complementary role for the tetraspanins CD37 and Tssc6 in cellular immunity. J Immunol. 2010;185:3158-66.

52. Kothapalli R, Bailey RD, Kusmartseva I, Mane S, Epling-Burnette PK, Loughran Jr TP. Constitutive expression of cytotoxic proteases and down-regulation of protease inhibitors in LGL leukemia. Int J Oncol. 2003;22:33-9.

53. Park JB, Lee CS, Jang J-H, Ghim J, Kim Y-J, You S, et al. Phospholipase signalling networks in cancer. Nat Rev Cancer. 2012;12:782-92.

54. Bartlett JMS, Thomas J, Ross DT, Seitz RS, Ring BZ, Beck RA, et al. Mammostrat as a tool to stratify breast cancer patients at risk of recurrence during endocrine therapy. Breast Cancer Res. 2010;12:R47.

55. McHugh BJ, Buttery R, Lad Y, Banks S, Haslett C, Sethi T. Integrin activation by Fam38A uses a novel mechanism of R-Ras targeting to the endoplasmic reticulum. J Cell Sci. 2010;123(Pt 1):51-61.

56. Tom Tang Y, Emtage P, Funk WD, Hu T, Arterburn M, Park EEJ, et al. TAFA: a novel secreted family with conserved cysteine residues and restricted expression in the brain. Genomics. 2004;83:727-34.

57. Oláh J, Vincze O, Virók D, Simon D, Bozsó Z, Tõkési N, et al. Interactions of pathological hallmark proteins: tubulin polymerization promoting protein/ p25, beta-amyloid, and alpha-synuclein. J Biol Chem. 2011;286:34088-100.

58. Ko HY, Lee DS, Kim S. Noninvasive imaging of microRNA124a-mediated repression of the chromosome 14 ORF 24 gene during neurogenesis. FEBS J. 2009;276:4854-65.

59. Tsapara A, Matter K, Balda MS. The heat-shock protein Apg-2 binds to the tight junction protein ZO-1 and regulates transcriptional activity of ZONAB. Mol Biol Cell. 2006;17:1322-30.

60. Han J-Y, Shin ES, Lee Y-S, Ghang HY, Kim S-Y, Hwang J-A, et al. A genome-wide association study for irinotecan-related severe toxicities in patients with advanced non-small-cell lung cancer. Pharmacogenomics J. 2013;13:417-22.

61. Poulogiannis G, Ichimura K, Hamoudi RA, Luo F, Leung SY, Yuen ST, et al. Prognostic relevance of DNA copy number changes in colorectal cancer. J Pathol. 2010;220:338-47.

62. Moreira F, Kiehl T-R, So K, Ajeawung NF, Honculada C, Gould P, et al. NPAS3 demonstrates features of a tumor suppressive role in driving the progression of Astrocytomas. Am J Pathol. 2011;179:462-76.

63. Stevanin G, Santorelli FM, Azzedine H, Coutinho P, Chomilier J, Denora PS, et al. Mutations in SPG11, encoding spatacsin, are a major cause of spastic paraplegia with thin corpus callosum. Nat Genet. 2007;39:366-72.

64. Chuang LSH, Ito K, Ito Y. RUNX family: Regulation and diversification of roles through interacting proteins. Int J Cancer. 2013;132:1260-71.

\section{Submit your next manuscript to BioMed Central and take full advantage of:}

- Convenient online submission

- Thorough peer review

- No space constraints or color figure charges

- Immediate publication on acceptance

- Inclusion in PubMed, CAS, Scopus and Google Scholar

- Research which is freely available for redistribution

Submit your manuscript at www.biomedcentral.com/submit 